\title{
Hotspot of Crimean-Congo Hemorrhagic Fever Virus Seropositivity in Wildlife, Northeastern Spain
}

Johan Espunyes, Oscar Cabezón, Lola Pailler-García, Andrea Dias-Alves, Lourdes Lobato-Bailón, Ignasi Marco, Maria P. Ribas, Pedro E. Encinosa-Guzmán, Marta Valldeperes, Sebastian Napp

We conducted a serosurvey for Crimean-Congo hemorrhagic fever virus antibodies in various wildlife species in Catalonia, northeastern Spain. We detected high seroprevalence in southern Catalonia, close to the Ebro Delta wetland, a key stopover for birds migrating from Africa. Our findings could indicate that competent virus vectors are present in the region.

Crimean-Congo hemorrhagic fever virus $(\mathrm{CCH}-$ FV) is an arthropodborne Orthonairovirus mainly transmitted by ticks. In humans, CCHFV infection can cause severe and even fatal Crimean-Congo hemorrhagic fever (CCHF) disease (1). CCHFV also can infect wild and domestic mammalian species, producing viremia but causing a predominantly asymptomatic disease and such species have a role in the maintenance of the virus in the environment (2).

$\mathrm{CCHFV}$ is endemic in Africa, Asia, and eastern Europe but has more recently emerged in southwestern Europe. In 2010, CCHFV was detected in central-western Spain in Hyalomma lusitanicum ticks collected from red deer (Cervus elaphus) (3). In 2016, 2 autochthonous human CCHF cases were reported in Spain, 1 likely contracted through tick bite and the other caused by nosocomial transmission (4). Since then, 6 other CCHF clinical cases, including a retrospectively identified case from 2013, have been reported in the country, all of which are suspected

Author affiliations: Universitat Autònoma de Barcelona, Bellaterra, Spain (J. Espunyes, O. Cabezón, A. Dias-Alves, L. Lobato-Bailón, I. Marco, M.P. Ribas, P.E. Encinosa-Guzmán, M. Valldeperes); Centre de Recerca en Sanitat Animal, Bellaterra (O. Cabezón, L. Pailler-García, S. Napp)

DOI: https://doi.org/10.3201/eid2709.211105 to be caused by infected ticks $(5,6)$. Further surveys on ticks $(7,8)$, and serologic studies in humans $(9)$ and animals (10) have shown evidence of CCHFV circulation in several areas of central and southwestern Spain. The high genetic variability of the CCHFV strains identified in Spain, including genotypes Africa III and IV and Europe V, are indicative of repeated introductions $(7,8)$. The area of CCHFV detection coincides with the region where the ecologic conditions are more favorable for the presence of $H$. marginatum and $H$. lusitanicum ticks, the main vectors of the disease. Neither of these species have been reported in northeastern Spain, but ecologic models predict the existence of areas suitable for $H$. marginatum (11). To evaluate possible CCHFV circulation in Catalonia, northeastern Spain, we conducted a serosurvey to detect CCHFV antibodies in different susceptible wild animal species.

\section{The Study}

Serum samples from different wildlife species were collected during 2014-2020 as part of routine wildlife surveillance in Catalonia from areas representing different ecosystems (Figures 1, 2). We tested for CCHFV antibodies in serum samples from 174 red deer, 84 Iberian ibexes (Capra pyrenaica), 79 roe deer (Capreolus capreolus), 35 European rabbits (Oryctolagus cuniculus), 156 wild boars (Sus scrofa), and 4 fallow deer (Dama dama) (Table 1). We used the CCHF Double Antigen Multi-species ELISA kit (IDvet, https:/ / www.id-vet.com), which has a sensitivity of $98.9 \%$ (95\% CI $96.8 \%-99.8 \%)$ and a specificity of $100 \%(95 \%$ CI $99.8 \%-100 \%)(12)$.

Because CCHFV might have been introduced in the region via ticks carried by migratory birds (3), we selected 226 samples from areas close to the 3 main 


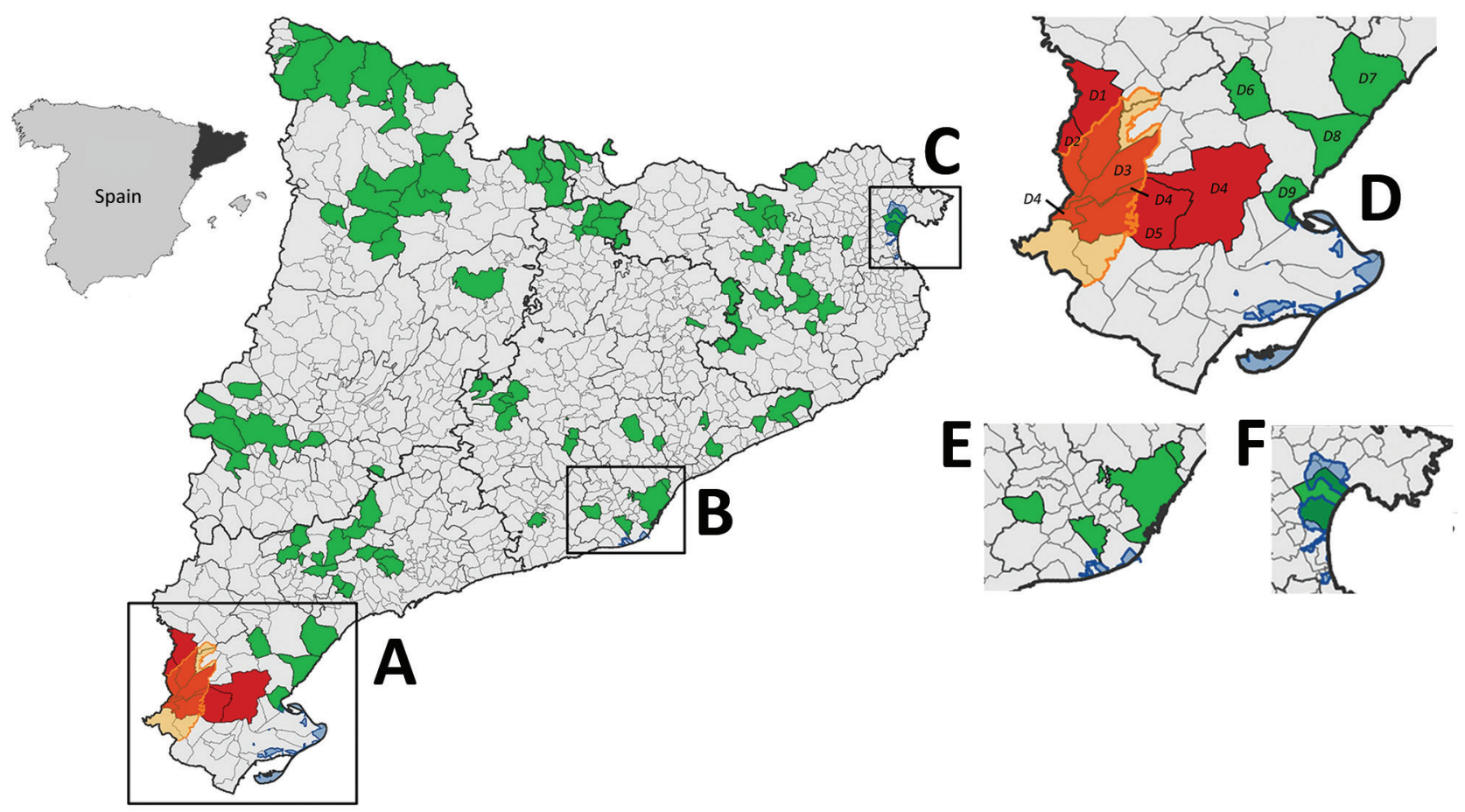

Figure 1. Distribution of areas sampled for detection of antibodies against Crimean-Congo hemorrhagic fever virus (CCHFV) in various species, Catalonia, northeastern Spain. Inset at left shows Catalonia (black) in northeastern Spain. Large map shows distribution of serosurveys throughout Catalonia: A) Ebro Delta; B) Llobregat Delta; C) Aiguamolls de l'Empordà; Enlarged areas represent regions with wetlands (blue shading), which are stopovers for migratory birds from Africa: D) Ebro Delta; E) Llobregat Delta; F) Aiguamolls de l'Empordà. Green shading indicates areas from which all samples were seronegative; red shading indicates $\geq 1$ sample was seropositive; gray shading indicates area was not sampled; yellow shading/outline indicates location of Ports de Tortosa-Beseit National Park. Additional details are provided on CCHFV hotspots in Ebro Delta (D), which are close to and overlap wetlands and Ports de Tortosa-Beseit Natural Park. Among regions in this area, animals tested (no. positive/no. tested) included the following: D1, Iberian ibexes 10/10, wild boar 4/21; D2, Iberian ibexes 17/17, roe deer 1/1, wild boar 1/3; D3, Iberian ibexes 3/3; D4, Iberian ibexes 8/8, European rabbit 0/2; D5, Iberian ibexes 28/28, European rabbit 0/2; D6, European rabbit 0/6; D7, roe deer 0/1; D8, European rabbit 0/1; and D9, European rabbit 0/1.

points of arrival of birds from Africa: the wetlands of the Ebro Delta $(\mathrm{n}=101)$; the Llobregat Delta $(\mathrm{n}=82)$, in close proximity to the urban area of Barcelona; and the Aiguamolls de $l^{\prime}$ Empordà $(n=43)$. The remaining 306 samples were collected from municipalities throughout Catalonia.

Of 532 samples tested, CCHFV antibodies were detected in 72 animals, including Iberian ibex $(66 / 84)$, roe deer $(1 / 79)$, and wild boar $(5 / 156)$ (Tables 1, 2). All 72 seropositive samples came from the same area in southern Catalonia, which includes 5 municipalities within or close to the Ports de Tortosa-Beseit Natural Park (Figure 1). This area is composed of rugged terrain, including canyons and ravines, and mainly is covered by a Mediterranean forest dominated by oaks, pines, and dense shrubland. This natural area is located a few kilometers from the Ebro Delta, one of the main wetlands in Spain and a key stopover for birds migrating from Africa to Europe. Thus, CCHFV introduction via infected ticks transported by migrating birds seems plausible.

The 66 Iberian ibexes tested in the affected area during 2017-2019, and 1/2 roe deer sampled in 2019, were CCHFV-positive, indicating high seroprevalence in the area since at least 2017. A 2018 serosurvey in wild ruminants also found a high seroprevalence $(79 \%)$ in some areas of central Spain known to have Hyalomma ticks but where CCHFV had not been detected previously (10). In contrast, of 24 wild boars sampled from affected municipalities during 2017-2020, only 5 (20.8\%) were seropositive. Reasons for the difference in seroprevalence between Iberian ibexes and wild boars are not clear and will require additional studies. One possible explanation would be that adult Hyalomma ticks feed preferentially on the family Bovidae (13); high seroprevalences frequently are observed in Spain among domestic goats (Capra aegagrus hircus), a closely related species (10). European rabbits tested 

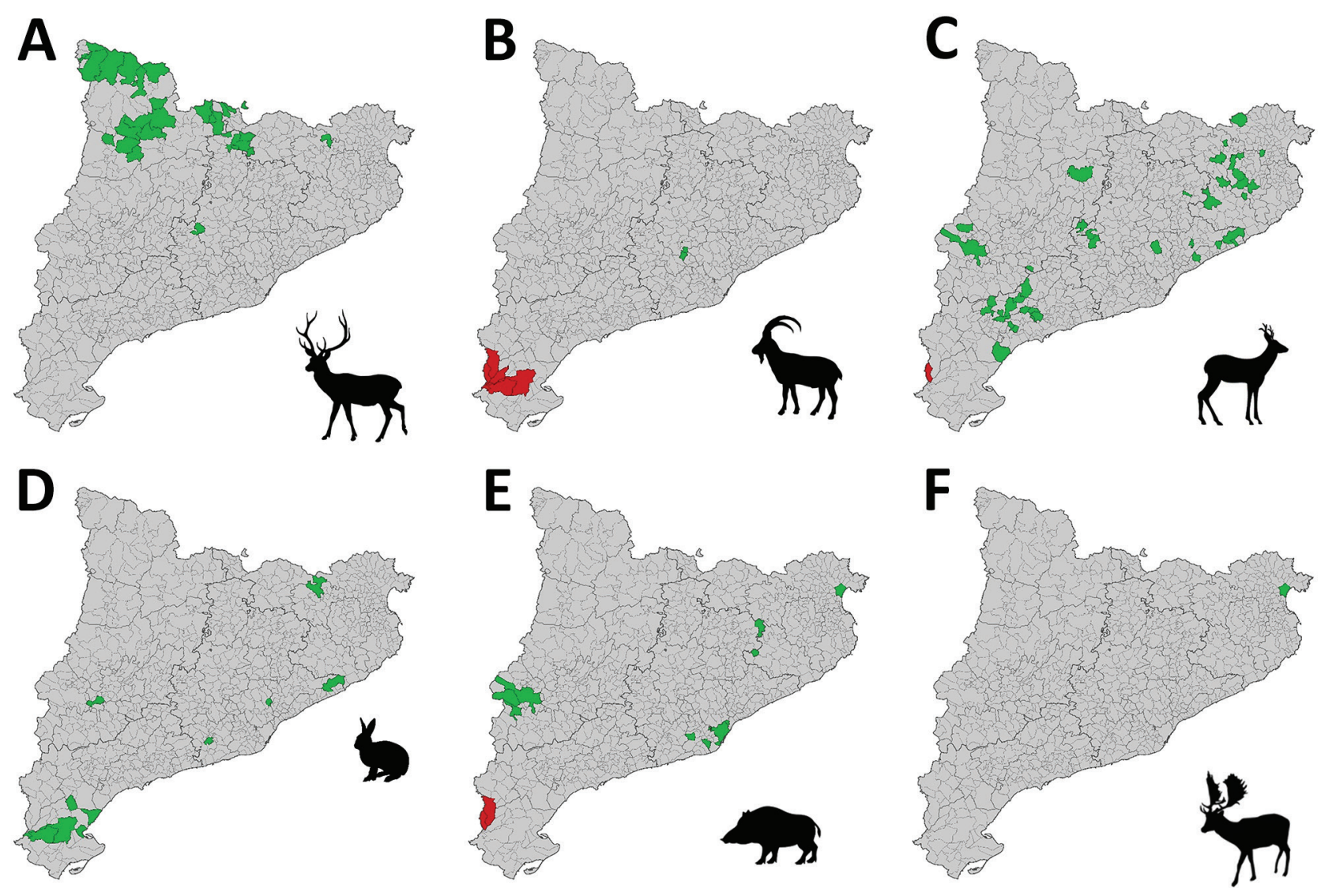

Figure 2. Distribution of areas sampled for detection of antibodies against Crimean-Congo hemorrhagic fever virus CCHFV by species, Catalonia, northeastern Spain. Green indicates all samples were seronegative; red indicates $\geq 1$ sample was seropositive; gray indicates areas not sampled. A) Red deer (Cervus elaphus); B) Iberian ibex (Capra pyrenaica); C) roe deer (Capreolus capreolus); D) European rabbit (Oryctolagus cuniculus); E) wild boar (Sus scrofa); F) fallow deer (Dama dama).

in the affected area were seronegative (Table 2); however, they were sampled in 2016 when CCHFV might not have been introduced or might have been at lower levels. No CCHFV antibodies were detected in red deer or fallow deer, but in the areas where they were sampled, seropositivity was not detected in any of the other susceptible species either (Figure 2).

\section{Conclusions}

Detection of CCHFV antibodies among animals in southern Catalonia implies the availability of competent vectors, most likely $H$. marginatum ticks; however, presence of $H$. marginatum ticks in the area and on the host species will need to be confirmed. The range of $H$. marginatum ticks is expanding in Europe; permanent populations have been reported in southern France (14). This expansion probably is influenced by the density of wild ungulates, from which adult $H$. marginatum ticks feed, and leporids, from which immature ticks feed. In Catalonia, increasing populations of rabbits and wild ungulates, including wild boar, roe deer, and fallow deer, have

\begin{tabular}{|c|c|c|c|c|c|c|}
\hline Species & $2014-2016$ & 2017 & 2018 & 2019 & 2020 & Total \\
\hline Red deer & $0 / 13(0 \%-28 \%)$ & $0 / 60(0 \%-1 \%)$ & $0 / 15(0 \%-3 \%)$ & $0 / 29(0 \%-15 \%)$ & $0 / 57(0 \%-8 \%)$ & $0 / 174(0 \%-3 \%)$ \\
\hline Iberian ibex & & $15 / 15(75 \%-100 \%)$ & $5 / 5$ & $46 / 46(90 \%-100 \%)$ & $0 / 18(0 \%-22 \%)$ & $66 / 84(68 \%-87 \%)$ \\
\hline Roe deer & & $0 / 1$ & $0 / 1$ & $1 / 59(0 \%-10 \%)$ & $0 / 18(0 \%-22 \%)$ & $1 / 79(0 \%-8 \%)$ \\
\hline $\begin{array}{l}\text { European } \\
\text { rabbit }\end{array}$ & $0 / 21(0 \%-19 \%)$ & $0 / 11(0 \%-32 \%)$ & $0 / 3$ & & & $0 / 35(0 \%-12 \%)$ \\
\hline $\begin{array}{l}\text { Wild boar } \\
\text { Fallow deer }\end{array}$ & & $1 / 87(0 \%-7 \%)$ & $3 / 3$ & 0 & $1 / 48(0 \%-13 \%)$ & $5 / 156(1 \%-8 \%)$ \\
\hline Total & $0 / 34(0 \%-13 \%)$ & $16 / 174(6 \%-15 \%)$ & $8 / 27(15 \%-50 \%)$ & $47 / 156(23 \%-38 \%)$ & $1 / 141(0 \%-5 \%)$ & $72 / 532(11 \%-17 \%)$ \\
\hline
\end{tabular}


Table 2. Distribution of samples tested for the presence of antibodies against CCHFV among various mammalian species, Ebro Delta area, Spain*

\begin{tabular}{|c|c|c|c|c|c|c|}
\hline Species & 2014-2016 & 2017 & 2018 & 2019 & 2020 & Total \\
\hline Iberian ibex & & $15 / 15(75 \%-100 \%)$ & $5 / 5$ & $46 / 46(90 \%-100 \%)$ & & $66 / 66(93 \%-100 \%)$ \\
\hline Roe deer & & & & $1 / 2$ & & $1 / 2$ \\
\hline European rabbit & $0 / 11(0 \%-32 \%)$ & & & & & $0 / 11(0 \%-32 \%)$ \\
\hline Wild boar & & $1 / 1$ & $3 / 3$ & $0 / 18(0 \%-22 \%)$ & $1 / 2$ & $5 / 24(8 \%-43 \%)$ \\
\hline Total & $0 / 11(0 \%-32 \%)$ & $16 / 16(76 \%-100 \%)$ & $8 / 8(60 \%-100 \%)$ & $47 / 66(59 \%-81 \%)$ & $1 / 2$ & $72 / 103(60 \%-78 \%)$ \\
\hline
\end{tabular}

required management measures to control their populations in recent years (15).

Besides southern Catalonia, samples from other areas evaluated in this study were seronegative. Whether seronegativity results from the absence of competent vectors or the absence of CCHFV is unclear, but defining seronegative and seropositive areas will be key in assessing risk for CCHFV transmission in the Mediterranean ecologic region. Further serosurveys to identify amplifying hosts and reservoirs of CCHFV in this ecologic region could help determine whether additional prevention measures against zoonotic transmission are needed in the area. Moreover, detecting the virus in hosts or vectors from the affected area and phylogenetic studies could clarify the origin of CCHFV in Catalonia. Risk for further introduction of $\mathrm{CCH}$ FV via migratory birds or expansion from the currently affected area to unaffected areas underscore the need for continued CCHF disease surveillance in Catalonia.

\section{Acknowledgments}

We thank the Generalitat de Catalunya for the support in the collection of samples.

\section{About the Author}

Dr. Espunyes is a researcher at the Wildlife Conservation Medicine Research Group (WildCoM), Departament de Medicina i Cirurgia Animals, Universitat Autònoma de Barcelona, Spain. His current research focuses on emerging diseases in wildlife species.

\section{References}

1. Bente DA, Forrester NL, Watts DM, McAuley AJ, Whitehouse CA, Bray M. Crimean-Congo hemorrhagic fever: history, epidemiology, pathogenesis, clinical syndrome and genetic diversity. Antiviral Res. 2013;100:15989. https:// doi.org/10.1016/j.antiviral.2013.07.006

2. Spengler JR, Estrada-Peña A, Garrison AR, Schmaljohn C, Spiropoulou CF, Bergeron É, et al. A chronological review of experimental infection studies of the role of wild animals and livestock in the maintenance and transmission of Crimean-Congo hemorrhagic fever virus. Antiviral Res. 2016;135:31-47. https://doi.org/10.1016/ j.antiviral.2016.09.013
3. Estrada-Peña A, Palomar AM, Santibáñez P, Sánchez N, Habela MA, Portillo A, et al. Crimean-Congo hemorrhagic fever virus in ticks, southwestern Europe, 2010. Emerg Infect Dis. 2012;18:179-80. https://doi.org/10.3201/eid1801.111040

4. Negredo A, de la Calle-Prieto F, Palencia-Herrejón E, Mora-Rillo M, Astray-Mochales J, Sánchez-Seco MP, et al.; Crimean Congo Hemorrhagic Fever@Madrid Working Group. Autochthonous Crimean-Congo Hemorrhagic fever in Spain. N Engl J Med. 2017;377:154-61. https://doi.org/10.1056/NEJMoa1615162

5. Portillo A, Palomar AM, Santibáñez P, Oteo JA. Epidemiological aspects of Crimean-Congo hemorrhagic fever in western Europe: what about the future? Microorganisms. 2021;9:649. https:// doi.org/10.3390/ microorganisms 9030649

6. Negredo A, Sánchez-Ledesma M, Llorente F, Pérez-Olmeda M, Belhassen-García M, González-Calle D, et al. Retrospective identification of early autochthonous case of Crimean-Congo hemorrhagic fever, Spain, 2013. Emerg Infect Dis. 2021;27:1754-6. https:/ / doi.org/10.3201/ eid2706.204643

7. Negredo A, Habela MÁ, Ramírez de Arellano E, Diez F, Lasala F, López P, et al. Survey of Crimean-Congo hemorrhagic fever enzootic focus, Spain, 2011-2015. Emerg Infect Dis. 2019;25:1177-84. https:/ /doi.org/10.3201/ eid2506.180877

8. Moraga-Fernández A, Ruiz-Fons F, Habela MA, Royo-Hernández L, Calero-Bernal R, Gortazar C, et al. Detection of new Crimean-Congo haemorrhagic fever virus genotypes in ticks feeding on deer and wild boar, Spain. Transbound Emerg Dis. 2021;68:993-1000. https://doi.org/ $10.1111 /$ tbed.13756

9. Monsalve Arteaga L, Muñoz Bellido JL, Vieira Lista MC, Vicente Santiago MB, Fernández Soto P, Bas I, et al. Crimean-Congo haemorrhagic fever (CCHF) virusspecific antibody detection in blood donors, Castile-León, Spain, summer 2017 and 2018. Euro Surveill. 2020;25:1900507. https://doi.org/10.2807/ 1560-7917.ES.2020.25.10.1900507

10. Ministry of Health, Consumption and Social Welfare. Report on the situation and evaluation of the risk of transmission of the Crimean-Congo hemorrhagic fever virus in Spain, July 2019 [in Spanish] [cited 2021 May 2]. https:/ / www.mscbs. gob.es/profesionales/saludPublica/ccayes/analisisituacion/ doc/ER_FHCC.pdf

11. Fernández-Ruiz N, Estrada-Peña A. Towards new horizons: climate trends in Europe increase the environmental suitability for permanent populations of Hyalomma marginatum (Ixodidae). Pathogens. 2021;10:95. https:/ / doi.org/ $10.3390 /$ pathogens 10020095

12. Sas MA, Comtet L, Donnet F, Mertens M, Vatansever Z, Tordo N, et al. A novel double-antigen sandwich ELISA for the species-independent detection of Crimean-Congo hemorrhagic fever virus-specific antibodies. Antiviral Res. 2018;151:24-6. https://doi.org/10.1016/ j.antiviral.2018.01.006 
13. Spengler JR, Estrada-Peña A. Host preferences support the prominent role of Hyalomma ticks in the ecology of Crimean-Congo hemorrhagic fever. PLoS Negl Trop Dis. 2018; 12:e0006248. https://doi.org/10.1371/journal.pntd.0006248

14. Vial L, Stachurski F, Leblond A, Huber K, Vourc'h G, René-Martellet $\mathrm{M}$, et al. Strong evidence for the presence of the tick Hyalomma marginatum Koch, 1844 in southern continental France. Ticks Tick Borne Dis. 2016;7:1162-7. https://doi.org/10.1016/j.ttbdis.2016.08.002

15. Department of Agriculture, Livestock, Fisheries and Food. Damage and risk prevention plan originated by hunting fauna (2017-2018) [in Catalan] [cited 2021 May 2]. http://agricultura.gencat.cat/web/.content/01departament/plans-programes-sectorials/enllacosdocuments/fitxers-binaris/pla-prevencio-danys-riscosoriginats-fauna-cinegetica-2017-2018.pdf

Address for correspondence: Oscar Cabezón, Wildlife Conservation Medicine Research Group (WildCoM), Departament de Medicina i Cirurgia Animals, Universitat Autònoma de Barcelona, 08193 Bellaterra, Spain; email: oscar.cabezon@uab.cat

\section{August 2020} Parasitic Infections

- Association of Dengue Virus and Leptospira Co-Infections with Malaria Severity

- US CDC Real-Time Reverse Transcription PCR Panel for Detection of Severe Acute Respiratory Syndrome Coronavirus 2

- Coronavirus Disease Outbreak in Call Center, South Korea

- Investigation and Serologic FollowUp of Contacts of an Early Confirmed Case-Patient with COVID-19, Washington, USA

- Characteristics and Outcomes of Coronavirus Disease Patients under Nonsurge Conditions, Northern California, USA, March-April 2020

- Tuberculosis in Internationally Displaced Children Resettling in Harris County, Texas, USA, 2010-2015

- Epidemiology of Legionnaires' Disease, Hong Kong, China, 2005-2015

- Rise in Babesiosis Cases, Pennsylvania, USA, 2005-2018

- Sporadic Creutzfeldt-Jakob Disease among Physicians, Germany, 1993-2018

- Analysis of MarketScan Data for Immunosuppressive Conditions and Hospitalizations for Acute Respiratory Illness, United States

- CrAssphage as a Novel Tool to Detect Human Fecal Contamination on Environmental Surfaces and Hands

- Evaluating the Effectiveness of Social Distancing Interventions to Delay or Flatten the Epidemic Curve of Coronavirus Disease

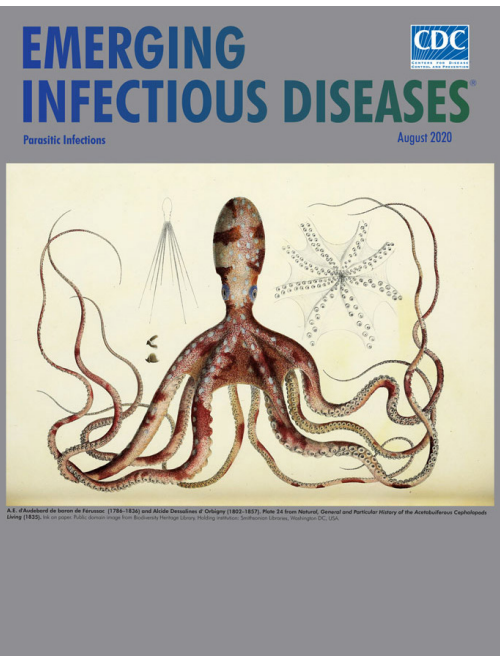

- Presence of Segmented Flavivirus Infections in North America

- Population Genomic Structure and Recent Evolution of Plasmodium knowlesi, Peninsular Malaysia

- Human Outbreak of Trichinellosis Caused by Trichinella papuae Nematodes, Central Kampong Thom Province, Cambodia

- Increased Sensitivity of Plasmodium falciparum to Artesunate/Amodiaquine Despite 14 Years as First-Line Malaria Treatment, Zanzibar

- Factors Associated with Prescription of Antimicrobial Drugs for Dogs and Cats, United Kingdom, 2014-2016

- Linezolid-Associated Neurologic Adverse Events in Patients with MultidrugResistant Tuberculosis, France
- Naturally Acquired Human Plasmodium cynomolgi and P. knowlesi Infections, Malaysian Borneo

- Characterizing Norovirus Transmission from Outbreak Data, United States

- Imported Monkeypox, Singapore

- Population-Based Estimates of Chronic Conditions Affecting Risk for Complications from Coronavirus Disease, United States

- Prolonged Persistence of SARS-CoV-2 RNA in Body Fluids

- Prognostic Value of Leukocytosis and Lymphopenia for Coronavirus Disease Severity

- SARS-CoV-2 Phylogenetic Analysis, Lazio Region, Italy, FebruaryMarch 2020

- Plasma-Derived Extracellular Vesicles as Potential Biomarkers in Heart Transplant Patient with Chronic Chagas Disease

- Spread of Multidrug-Resistant Bacteria by Moth Flies from Hospital Waste Water System

- Disseminated Echinococcus multilocularis Infection without Liver Involvement in Child, Canada, 2018

- Canine Dracunculus Nematode Infection, Toledo, Spain

- Leishmania donovani Infection with Atypical Cutaneous Manifestations, Himachal Pradesh, India, 2014-2018

- Doxycycline and Sitafloxacin Combination Therapy for Treating Highly Resistant Mycoplasma genitalium

\section{EMERGING} INFECTIOUS DISEASES 